\title{
Treatment of nonalcoholic steatohepatitis with probiotics. A proof-of-concept study
}

\author{
Vincent Wai-Sun Wong, ${ }^{*}, * *$ Grace Lai-Hung Wong, ${ }^{*}, * *$ Angel Mei-Ling Chim, ${ }^{*}, * *$ \\ Winnie Chiu-Wing Chu, *** David Ka-Wai Yeung, **** Kevin Chi-To Li,* Henry Lik-Yuen Chan*,** \\ ${ }^{*}$ Department of Medicine and Therapeutics. ${ }^{* *}$ Institute of Digestive Disease. ${ }^{* *}$ Department of Imaging and Interventional Radiology. \\ ${ }_{* \star * \star}$ Department of Clinical Oncology, The Chinese University of Hong Kong, Hong Kong.
}

\section{ABSTRACT}

Background. Probiotics have profound effect on nonalcoholic steatohepatitis (NASH) in animal models. We aimed to test the hypothesis that probiotics treatment was superior to usual care in reducing liver fat in NASH patients. Material and methods. Patients with histology-proven NASH were randomized to receive probiotics $(n=10)$ or usual care $(n=10)$ for 6 months. The Lepicol probiotic formula contained Lactobacillus plantarum, Lactobacillus deslbrueckii, Lactobacillus acidophilus, Lactobacillus rhamnosus and Bifidobacterium bifidum. The primary endpoint was change in intrahepatic triglyceride content (IHTG), as measured by proton-magnetic resonance spectroscopy, from baseline to month 6 . Secondary endpoints included changes in liver biochemistry and metabolic profile. Results. IHTG decreased from $22.6 \pm 8.2 \%$ to $14.9 \pm$ $7.0 \%$ in the probiotic group $(P=0.034)$ but remained static in the usual care group $(16.9 \pm 6.1 \%$ to 16.0 $\pm 6.6 \% ; P=0.55)$. Six subjects in the probiotic group had IHTG reduced by more than $30 \%$ from baseline, compared to 2 subjects in the usual care group $(P=0.17)$. The probiotic group also had greater reduction in serum aspartate aminotransferase level $(P=0.008)$. On the other hand, the use of probiotics was not associated with changes in body mass index, waist circumference, glucose and lipid levels. Conclusions. Probiotics treatment may reduce liver fat and AST level in NASH patients. The therapeutic potential of probiotics in NASH should be tested in larger studies.

Key words. Fatty liver. Obesity. Randomized controlled trial. Prebiotics.

\section{INTRODUCTION}

Nonalcoholic fatty liver disease (NAFLD) is one of the most common chronic liver diseases worldwide. ${ }^{1}$ Its active form, nonalcoholic steatohepatitis (NASH), may progress to cirrhosis and hepatocellular carcinoma. ${ }^{2-5}$ At present, there is no registered drug for the treatment of NASH. While lifestyle management is often advocated, it is difficult to maintain. ${ }^{6}$ It is important to explore new treatment strategies.

The human body contains up to 100 trillion bacterial cells, most of which reside in the gut. Gut microbes interact closely with the human body, and

Correspondence and reprint request: Professor Henry Chan, M.D.

Department of Medicine and Therapeutics, 9/ F, Prince of Wales Hospital.

30-32 Ngan Shing Street, Shatin, Hong Kong.

Tel: +852 26323593. Fax: +85226373852

E-mail: hlychan@cuhk.edu.hk have substantial effect on energy harvest from food, systemic inflammation and innate immunity. ${ }^{7,8} \mathrm{In}$ animal and human studies, altered gut microbiota is associated with obesity. ${ }^{9,10}$ In addition, NASH patients have increased gut permeability and high blood levels of bacterial endotoxin, which results in liver injury. ${ }^{11} \mathrm{In}$ a recent report, changes in the fecal composition of Gamma proteobacteria and Erysipelotrichi were observed in 15 women with fatty liver induced by choline-deficient diet. ${ }^{12}$ These data suggest that gut microbiota may be involved in the pathogenesis of NASH.

In animal studies, probiotics have profound effect on NASH. ${ }^{13-15}$ Probiotics lead to reduced activity of Jun $\mathrm{N}$-terminal kinase and nuclear factor $\kappa b .{ }^{13} \mathrm{It}$ also increases the number of natural killer $\mathrm{T}$ cells in the liver. ${ }^{14}$ Although another animal study failed to demonstrate an effect on hepatic steatosis and necroinflammation, probiotics prevented liver fibrosis through changes in the expression of transforming growth factor beta. ${ }^{16}$ It is unclear if probiotics are efficacious in human NASH patients. 
Furthermore, prebiotics are non-digestible carbohydrates that alter the composition and activity of the gut microbiota. ${ }^{17}$ It reduces de novo fatty acid synthesis, insulin resistance and metabolic endotoxemia. ${ }^{18-20}$ Although there has been no trials on $\mathrm{NASH}$, prebiotics lower cholesterol and triglyceride levels in humans. ${ }^{21}$

In this study, we aimed to test the hypothesis that probiotics treatment was superior to usual care in reducing liver fat in patients with NASH.

\section{MATERIAL AND METHODS}

\section{Study design}

This was an open-label, randomized controlled trial in patients with histology-proven NASH. Consecutive patients attending the hepatology clinic of the Prince of Wales Hospital, Hong Kong were screened. Eligible patients were randomized in 1:1 ratio to receive probiotic treatment or usual care for 6 months. Randomization was carried out through the use of computer-generated list of random numbers in blocks of 4 . Treatment assignment was kept in sealed envelopes until the time of randomization. All study medications were dispensed by a research nurse. Compliance with the study medication was calculated from the unused sachets of study medications at each visit. The study protocol was approved by the ethics committee of The Chinese University of Hong Kong. All subjects gave informed written consent. While the subjects in both groups did not receive monetary compensation, they received free clinical care and medical tests. The study was registered at ClinicalTrials.gov (NCT00870012).

\section{Subjects}

Patients aged 18 to 70 years had histology-proven $\mathrm{NASH}$, defined as hepatic steatosis of more than 5\% and the presence of either hepatocyte ballooning or intralobular hepatocyte necrosis on a liver biopsy performed within 6 months before inclusion. Histological scoring was also performed according to the NASH Clinical Research Network system. ${ }^{22}$ These patients had elevated alanine aminotransferase (ALT) level of over $30 \mathrm{IU} / \mathrm{L}$ in men and $19 \mathrm{IU} / \mathrm{L}$ in women. We excluded patients with positive hepatitis $\mathrm{B}$ surface antigen, antibody against hepatitis $\mathrm{C}$ virus, or anti-nuclear antibody titer above $1 / 160$; significant alcohol consumption (over $20 \mathrm{~g}$ per day in men or $10 \mathrm{~g}$ per day in women); ALT above 10 times the upper limit of normal; liver decompensation or malignancy; or consumption of systemic corticosteroids or methotrexate in the last 6 months.

\section{Study treatment and follow-up}

At baseline, all NASH patients received lifestyle advice from an investigator. They were instructed to lose weight, reduce fat intake, and exercise at least 3 times per week. Patients randomized to the probiotic group also received one sachet of Lepicol probiotic and prebiotic formula (Healthy Bowels Company Ltd, Birmingham, UK) twice a day for 6 months. The formula contained 5 probiotic cultures including Lactobacillus plantarum (ATCC 14917), Lactobacillus delbrueckii ssp. bulgaricus (ATCC 11842), Lactobacillus acidophilus (ATCC 4356), Lactobacillus rhamnosus (ATCC 7469) and Bifidobacterium bifidum (ATCC 29521). Each $10 \mathrm{~g}$ sachet contained 200 million probiotic cultures. Other ingredients of the formula included $3 \mathrm{~g}$ of fructo-oligosaccharides (prebiotics), cellulose, magnesium stearate, silica and milk.

A research nurse contacted each patient by phone at week 4 to identify new symptoms. Thereafter, the patients were followed up at months 3 and 6 . At each visit, new symptoms and concomitant drugs were recorded. Body weight, body height, waist circumference and blood pressure were measured. Waist circumference was measured at a level midway between the lower rib margin and iliac crest with the tape all around the body in the horizontal position. Body mass index (BMI) was calculated as body weight (kg) divided by body height $(\mathrm{m})$ squared. Blood was taken for liver biochemistry, glucose and lipids after fasting for at least $8 \mathrm{~h}$. All biochemical tests were performed using the cobas ${ }^{\circledR} 8000$ modular analyzer (Roche Diagnostics, West Sussex, England).

\section{Proton-magnetic resonance spectroscopy}

Intrahepatic triglyceride content (IHTG) was measured at baseline and month 6 by proton-magnetic resonance spectroscopy using the whole body 3 Tesla scanner (Philips Healthcare, Best, the Netherlands). Whole-body 3.0T scanner with a single voxel point-resolved spectroscopy sequence and an echo time of $40 \mathrm{~ms}$ and repetition time of $5,000 \mathrm{~ms}$ was used. A survey scan was first performed in the abdominal region to help positioning a volume measuring $20(\mathrm{AP}) \times 15(\mathrm{RL}) \times 40(\mathrm{FH}) \mathrm{mm}$ within the liver. The scanner's built-in body coil was used for both signal transmission and reception. No-water- 
suppressed spectra was acquired using 32 signal averages and the data were exported for offline spectral analysis. Water $(4.65 \mathrm{ppm})$ and lipid (1.3 ppm) peak amplitudes were measured to determine vertebral marrow fat content, which was defined as the relative fat signal amplitude in terms of a percentage of the total signal amplitude (water and fat) and calculated according to the following equation:

$$
\text { Fat content }=[\text { Ifat } /(\text { Ifat }+ \text { Iwater })] \times 100 .
$$

Where Ifat and Iwater are the peak amplitudes of fat and water, respectively. Radiologists performing spectral analysis were blinded to the treatment assignment.

\section{Study endpoints}

The primary endpoint was the reduction in IHTG from baseline to month 6 as measured by proton-magnetic resonance spectroscopy. Secondary endpoints included changes in ALT, aspartate aminotransferase (AST), BMI, fasting glucose, lipids and liver stiffness measurement by transient elastography. Liver stiffness measurement was performed using the Fibroscan machine (Echosens, Paris, France). The procedure was performed according to the manufacturer's instructions as described previously. ${ }^{23,24}$ Liver stiffness measurements were considered reliable only if 10 successful acquisitions were obtained, the success rate was above $60 \%$, and the interquartile range (IQR)-tomedian ratio was below 0.3 .

\section{Statistical analysis}

Continuous variables were expressed in mean \pm standard deviation, or median (IQR) if they were not in normal distribution. Continuous variables were compared between the two treatment arms using unpaired $t$ test or Mann-Whitney $\mathrm{U}$ test. Categorical variables were compared using $\chi^{2}$ test or Fisher exact test as appropriate. The change in IHTG within group was tested using paired t test. Statistical significance was taken as a two-sided $\mathrm{P}$ value of $<0.05$.

This was a pilot study. We considered a reduction of IHTG by $30 \%$ to be clinically significant. Assuming that the reduction of IHTG was $30 \%$ in the probiotic group and $10 \%$ in the usual care group with a standard deviation of $15 \%$, a sample size of 9 patients per arm would achieve $80 \%$ power to detect the difference at a $5 \%$ significance level. Allowing a $10 \%$ dropout rate, a total of 20 patients were required.

\section{RESULTS}

From March to September 2009, 20 patients were enrolled and underwent randomization (Figure 1). Ten patients were randomized to the probiotic group and ten patients were assigned to the usual care group. The two groups were well matched, although there was a trend that patients in the usual care group were less obese (Table 1). None of the patients were lost to follow-up. All patients in the probiotic group took at least $80 \%$ of the study medication.

\section{Changes in liver fat}

At month 6, IHTG by proton-magnetic resonance spectroscopy decreased from $22.6 \pm 8.2 \%$ to $14.9 \pm$ $7.0 \%$ in the probiotic group (mean reduction $7.7 \%$; $95 \%$ confidence interval $0.7 \%, 14.7 \% ; \mathrm{P}=0.034$ ). In contrast, IHTG remained static in the usual care group at $16.9 \pm 6.1 \%$ at baseline and $16.0 \pm 6.6 \%$ at month 6 (mean reduction $1.0 \%$; 95\% confidence interval $-2.5 \%, 4.5 \% ; \mathrm{P}=0.55$ ). The probiotic group tended to have greater reduction in IHTG (Table 2). Overall, 8 subjects in the probiotic group and 4 subjects in the usual care group had reduced IHTG at month $6(\mathrm{P}=0.17)$ (Figure 2$)$. Six subjects in the probiotic group had IHTG reduced by more than $30 \%$ from baseline, compared to 2 subjects in the usual care group $(\mathrm{P}=0.17)$.

\section{Biochemical and metabolic response}

Similarly, the probiotics group had greater reduction in AST level than the usual care group (Table 2 ). On the other hand, there was no significant effect of probiotic treatment on the body mass index, waist circumference, glucose and lipid levels, and liver stiffness.

\section{Adverse events}

Adverse events occurred in 4 patients in each treatment group (Table 3). All reported adverse events were minor and did not differ between treatment groups.

\section{DISCUSSION}

In this proof-of-concept study in human patients with histology-proven NASH, probiotics treatment for 6 months resulted in reduction in liver fat and AST level. 


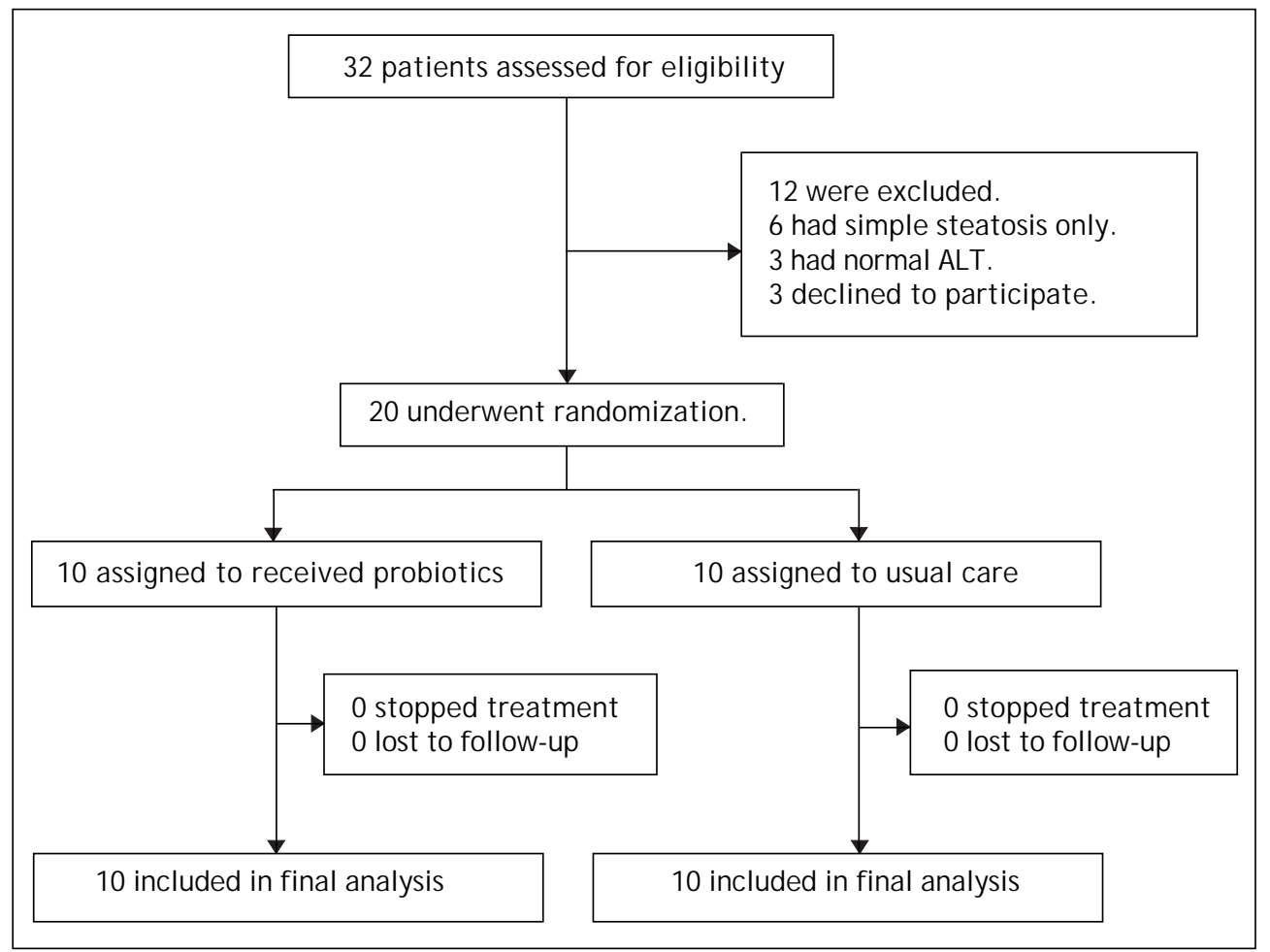

Figure 1. Screening, randomization and follow-up of study subjects.

Table 1. Baseline characteristics of subjects with and without NASH.

\begin{tabular}{|c|c|c|}
\hline Characteristics & Probiotic group $(n=10)$ & Usual care group $(n=10)$ \\
\hline Age (years). & $42 \pm 9$ & $55 \pm 9$ \\
\hline Male:Female. & $8: 2$ & $5: 5$ \\
\hline BMI $\left(\mathrm{kg} / \mathrm{m}^{2}\right)$. & $30.2 \pm 5.0$ & $28.7 \pm 5.7$ \\
\hline Waist circumference $(\mathrm{cm})$. & $101 \pm 10$ & $97 \pm 14$ \\
\hline ALT (IU/L). & $96 \pm 75$ & $72 \pm 30$ \\
\hline AST (IU/L). & $50 \pm 25$ & $38 \pm 15$ \\
\hline Fasting glucose (mmol/ L). & $6.1 \pm 1.4$ & $6.2 \pm 1.0$ \\
\hline Total cholesterol (mmol/ L). & $4.8 \pm 0.8$ & $5.0 \pm 1.0$ \\
\hline HDL-cholesterol ( $\mathrm{mmol} / \mathrm{L})$. & $1.2 \pm 0.2$ & $1.3 \pm 0.4$ \\
\hline LDL-cholesterol (mmol/ L). & $2.6 \pm 0.6$ & $2.8 \pm 0.9$ \\
\hline Triglycerides $(\mathrm{mmol} / \mathrm{L})$. & $2.7(1.4,3.1)$ & $1.8(1.3,2.6)$ \\
\hline Diabetes $(n)$. & 3 & 4 \\
\hline Hypertension (n). & 5 & 7 \\
\hline Sulfonylurea (n). & 2 & 1 \\
\hline Metformin (n). & 3 & 4 \\
\hline Thiazolidinedione (n). & 0 & 0 \\
\hline Insulin (n). & 1 & 0 \\
\hline Steatosis grade $(1 / 2 / 3)$. & $0 / 5 / 5$ & $1 / 5 / 4$ \\
\hline Lobular inflammation $(0 / 1 / 2 / 3)$. & $3 / 6 / 1 / 0$ & $2 / 8 / 0 / 0$ \\
\hline Ballooning (0/ 1/ 2). & $0 / 10 / 0$ & $0 / 9 / 1$ \\
\hline Fibrosis stage $(0 / 1 / 2 / 3 / 4)$. & $3 / 5 / 1 / 1 / 0$ & $3 / 4 / 0 / 1 / 2$ \\
\hline NAFLD activity score. & $4(4,5)$ & $5(4,5)$ \\
\hline Intrahepatic triglyceride content (\%). & $22.6 \pm 8.2$ & $16.9 \pm 6.1$ \\
\hline Liver stiffness ( $\mathrm{kPa})$. & $8.0(6.2,12.0)$ & $8.8(5.2,11.5)$ \\
\hline
\end{tabular}

Continuous variables were expressed as mean \pm standard deviation or median (interquartile range). ALT: alanine aminotransferase. BMl: body mass index. HDL: high density lipoprotein. LDL: Iow density lipoprotein. NASH: nonalcoholic steatohepatitis. 
Table 2. Changes in hepatic and metabolic parameters between baseline and month 6 .

\begin{tabular}{|c|c|c|c|}
\hline Parameters & Probiotic group & Usual care group & P* \\
\hline \multicolumn{4}{|l|}{ Hepatic triglyceride content. } \\
\hline Absolute percentage. & $-7.7 \pm 9.8$ & $-0.9 \pm 4.9$ & 0.065 \\
\hline Percentage of baseline value. & $-29 \pm 35$ & $-7.7 \pm 9.8$ & 0.083 \\
\hline BMI $\left(\mathrm{kg} / \mathrm{m}^{2}\right)$ & $-1.0 \pm 2.3$ & $-0.5 \pm 1.1$ & 0.57 \\
\hline Waist circumference $(\mathrm{cm})$. & $-2 \pm 6$ & $1 \pm 4$ & 0.34 \\
\hline \multicolumn{4}{|l|}{$\operatorname{ALT}(I U / L)$} \\
\hline Absolute change. & $-26 \pm 91$ & $2 \pm 41$ & 0.39 \\
\hline Percentage of baseline value. & $-13 \pm 56$ & $15 \pm 59$ & 0.28 \\
\hline \multicolumn{4}{|l|}{ AST (IU/L). } \\
\hline Absolute change. & $-13 \pm 31$ & $23 \pm 32$ & 0.021 \\
\hline Percentage of baseline value. & $-16 \pm 39$ & $37 \pm 40$ & 0.008 \\
\hline Fasting glucose (mmol/ L). & $0.8 \pm 2.9$ & $0.2 \pm 0.7$ & 0.51 \\
\hline Total cholesterol (mmol/ L). & $0 \pm 0.4$ & $0.2 \pm 0.5$ & 0.39 \\
\hline HDL-cholesterol (mmol/ L). & $0 \pm 0.1$ & $0.1 \pm 0.1$ & 0.14 \\
\hline LDL-cholesterol (mmol/ L). & $0.1 \pm 0.6$ & $0.3 \pm 0.5$ & 0.50 \\
\hline Triglycerides (mmol/ L). & $-0.1(-0.8,0.3)$ & $-0.3(-0.5,-0.1)$ & 0.68 \\
\hline Liver stiffness (kPa). & $-0.7(-2.0,0)$ & $0(-2.5,0.9)$ & 0.56 \\
\hline
\end{tabular}

Continuous variables were expressed as mean \pm standard deviation or median (interquartile range). ${ }^{*}$ Comparison between groups was performed using unpaired $t$ test or Mann-Whitney U test. ALT: alanine aminotransferase. AST: aspartate aminotransferase. BMI: body mass index. HDL: high density lipoprotein. LDL: low density lipoprotein.
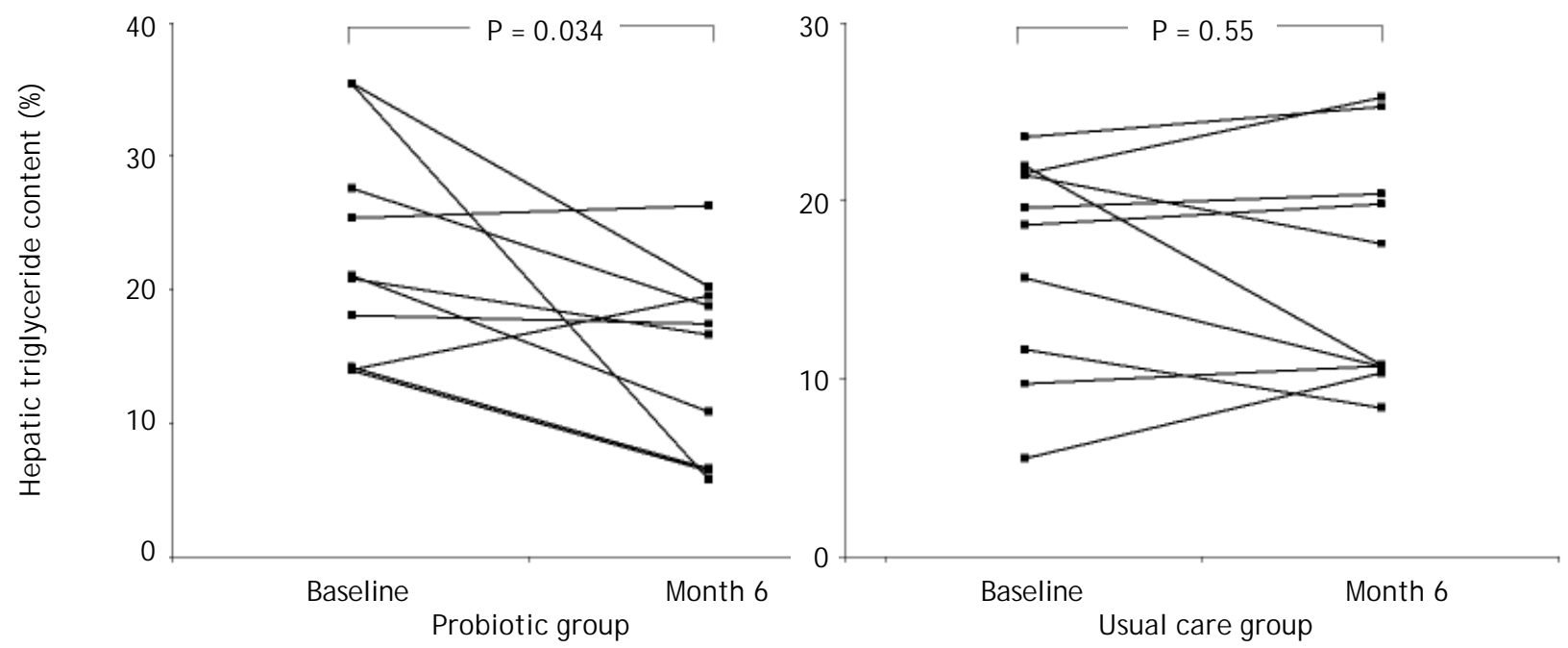

Figure 2. Intrahepatic triglyceride content at baseline and month 6 .

Table 3. Adverse events.

\begin{tabular}{lcc}
\hline Event & Probiotic group $(\mathrm{n}=10)$ & Usual care group $(\mathrm{n}=10)$ \\
\hline Dyspepsia & 2 & 1 \\
Musculoskeletal pain & 2 & 1 \\
Cough & 0 & 1 \\
Skin rash & 0 & 1 \\
\hline
\end{tabular}


The effect of probiotics on NASH has been tested in a number of animal studies. In general, probiotics treatment leads to improvement in hepatic steatosis and necroinflammation. ${ }^{13-15,25,26}$ On the other hand, the effect of probiotics in human NASH patients has not been adequately tested. A non-randomized study on the use of probiotic VSL\# 3 for 3 months in patients with different liver diseases (22 NAFLD, 20 alcoholic cirrhosis and 36 chronic hepatitis C) showed improved plasma levels of malondialdehyde, 4-hydroxynonenal and S-nitrosothiols. ${ }^{27}$ Recently, a randomized controlled trial of 28 patients with biopsy-proven NAFLD showed that a mixture of Lactobacillus bulgaricus and Streptococcus thermophilus for 3 months led to greater reduction in ALT, AST and gamma-glutamyl transferase levels. ${ }^{28}$ In our study, probiotics treatment with four strains of Lactobabillus and Bifidobacterium resulted in significant reduction in IHTG and AST level in patients with biopsy-proven NASH. IHTG by proton-magnetic resonance spectroscopy is currently one of the most accurate non-invasive tests for liver fat. ${ }^{1,29}$ These encouraging results provide foundation for further evaluation of probiotics treatment in larger studies using histological endpoints.

At present, the mechanism of action of probiotics in the treatment of NASH is incompletely understood. In murine models of obesity and fatty liver, probiotics reduce the expression of proinflammatory cytokines and modulate the activity of natural killer $\mathrm{T}$ cells in the liver. ${ }^{13-15,25}$ Interestingly, several strains of probiotics also ameliorate gut inflammation in the dextran sulfate sodium colitis model. ${ }^{30,31}$ Along the same line, the bacterial product lipopolysaccharide is increased in the portal blood of animals with colitis. ${ }^{32}$ In return, this promotes hepatic necroinflammation and fibrosis. Furthermore, NASH patients have increased small bowel bacterial overgrowth and higher blood level of endotoxin. ${ }^{11}$ In animal models, inflammasome-mediated dysbiosis also regulates progression of NAFLD through influx of toll-like receptors 4 and 9 in the portal circulation and overexpression of tumor necrosis factor-alpha. ${ }^{33}$ These data suggest the close relationship between gut bacteria and the pathogenesis of NASH, and these mechanisms may be modulated by the use of probiotics.

Our study has a few limitations. First, the sample size was relatively small. However, the consistent improvements in IHTG and AST level support further investigation in this area. Second, the results cannot be directly extrapolated to other probiotic formulae. Future studies are required to identify the roles and actions of individual bacterial strains.

In conclusion, probiotics treatment may reduce liver fat and AST level in NASH patients. The therapeutic potential of probiotics in NASH should be tested in larger studies.

\section{ABBREVIATIONS}

- ALT: alanine aminotransferase.

- AST: aspartate aminotransferase.

- BMI: body mass index.

- IHTG: intrahepatic triglyceride content.

- IQR: interquartile range.

- NAFLD: nonalcoholic fatty liver disease.

- NASH: nonalcoholic steatohepatitis.

\section{FINANCIAL SUPPORT}

The work described in this paper was partially supported by the direct grant of The Chinese University of Hong Kong (Ref 2010.1.042).

\section{COMPETING INTERESTS}

None declared.

\section{REFERENCES}

1. Wong VW, Chu WC, Wong GL, Chan RS, Chim AM, Ong A, Yeung DK, et al. Prevalence of non-alcoholic fatty liver disease and advanced fibrosis in Hong Kong Chinese: a population study using proton-magnetic resonance spectroscopy and transient elastography. Gut 2012; 61: 409-15.

2. Wong VW, Wong GL, Choi PC, Chan AW, Li MK, Chan HY, Chim AM, et al. Disease progression of non-alcoholic fatty liver disease: a prospective study with paired liver biopsies at 3 years. Gut 2010; 59: 969-74.

3. Ascha MS, Hanouneh IA, Lopez R, Tamimi TA, Feldstein AF, Zein NN. The incidence and risk factors of hepatocellular carcinoma in patients with nonalcoholic steatohepatitis. Hepatology 2010; 51: 1972-8.

4. Bhala N, Angulo P, van der Poorten D, Lee E, Hui JM, Saracco $G$, Adams $L A$, et al. The natural history of nonalcoholic fatty liver disease with advanced fibrosis or cirrhosis: an international collaborative study. Hepatology 2011; 54: 1208-16.

5. Wong VW, Chan HL. Prevention of hepatocellular carcinoma: a concise review of contemporary issues. Ann Hepatol 2012; 11: 284-93.

6. Rodriguez-Hernandez $H$, Cervantes-Huerta $M$, RodriguezMoran M, Guerrero-Romero F. Decrease of aminotransferase levels in obese women is related to body weight reduction, irrespective of type of diet. Ann Hepatol 2011; 10: 486-92.

7. Turnbaugh PJ, Ley RE, Mahowald MA, Magrini V, Mardis ER, Gordon JI. An obesity-associated gut microbiome with increased capacity for energy harvest. Nature 2006; 444: 1027-31. 
8. Qin J, Li R, Raes J, Arumugam M, Burgdorf KS, Manichanh $C$, Nielsen $T$, et al. A human gut microbial gene catalogue established by metagenomic sequencing. Nature 2010; 464: 59-65.

9. Ley RE, Turnbaugh PJ, Klein S, Gordon JI. Microbial ecology: human gut microbes associated with obesity. Nature 2006; 444: 1022-3.

10. Turnbaugh PJ, Hamady M, Yatsunenko T, Cantarel BL, Duncan $A$, Ley $R E$, Sogin $M L$, et al. A core gut microbiome in obese and lean twins. Nature 2009; 457: 480-4.

11. Miele L, Valenza V, La Torre G, Montalto M, Cammarota G, Ricci $R$, Masciana $R$, et al. Increased intestinal permeability and tight junction alterations in nonalcoholic fatty liver disease. Hepatology 2009; 49: 1877-87.

12. Spencer MD, Hamp TJ, Reid RW, Fischer LM, Zeisel SH, Fodor AA. Association between composition of the human gastrointestinal microbiome and development of fatty liver with choline deficiency. Gastroenterology 2011; 140: $976-86$.

13. Li Z, Yang S, Lin H, Huang J, Watkins PA, Moser AB, Desimone $C$, et al. Probiotics and antibodies to TNF inhibit inflammatory activity and improve nonalcoholic fatty liver disease. Hepatology 2003; 37: 343-50.

14. Ma X, Hua J, Li Z. Probiotics improve high fat diet-induced hepatic steatosis and insulin resistance by increasing hepatic NKT cells. J Hepatol 2008; 49: 821-30.

15. Esposito E, lacono A, Bianco G, Autore G, Cuzzocrea S, Vajro $P$, Canani RB, et al. Probiotics reduce the inflammatory response induced by a high-fat diet in the liver of young rats. J Nutr 2009; 139: 905-11.

16. Velayudham A, Dolganiuc A, Ellis M, Petrasek J, Kodys K, Mandrekar P, Szabo G. VSL\#3 probiotic treatment attenuates fibrosis without changes in steatohepatitis in a diet-induced nonalcoholic steatohepatitis model in mice. Hepatology 2009; 49: 989-97.

17. Parnell J A, Raman M, Rioux KP, Reimer RA. The potential role of prebiotic fibre for treatment and management of non-alcoholic fatty liver disease and associated obesity and insulin resistance. Liver Int 2012; 32: 701-11.

18. Delzenne NM, Williams CM. Prebiotics and lipid metabolism. Curr Opin Lipidol 2002; 13: 61-7.

19. Cani PD, Neyrinck AM, Fava F, Knauf C, Burcelin RG, Tuohy KM, Gibson GR, et al. Selective increases of bifidobacteria in gut microflora improve high-fat-diet-induced diabetes in mice through a mechanism associated with endotoxaemia. Diabetologia 2007; 50: 2374-83.

20. Cani PD, Possemiers S, Van de Wiele T, Guiot Y, Everard A, Rottier 0 , Geurts $L$, et al. Changes in gut microbiota control inflammation in obese mice through a mechanism involving GLP-2-driven improvement of gut permeability. Gut 2009; 58: 1091-103.

21. Forcheron F, Cachefo A, Thevenon S, Pinteur C, Beylot M. Mechanisms of the triglyceride- and cholesterol-lowering effect of fenofibrate in hyperlipidemic type 2 diabetic patients. Diabetes 2002; 51: 3486-91.

22. Kleiner DE, Brunt EM, Van Natta M, Behling C, Contos MJ, Cummings OW, Ferrell LD, et al. Design and validation of a histological scoring system for nonalcoholic fatty liver disease. Hepatology 2005; 41: 1313-21.

23. Wong VW, Vergniol J, Wong GL, Foucher J, Chan HL, Le Bail B, Choi PC, et al. Diagnosis of fibrosis and cirrhosis using liver stiffness measurement in nonalcoholic fatty liver disease. Hepatology 2010; 51: 454-62.

24. Abenavoli L, Beaugrand M. Transient elastography in nonalcoholic fatty liver disease. Ann Hepatol 2012; 11: 172-8.

25. Nardone G, Compare D, Liguori E, Di Mauro V, Rocco A, Barone $M$, Napoli $A$, et al. Protective effects of Lactobacillus paracasei $F 19$ in a rat model of oxidative and metabolic hepatic injury. Am J Physiol Gastrointest Liver Physiol 2010; 299: G669-G676.

26. Rosberg-Cody E, Stanton C, O'Mahony L, Wall R, Shanahan F, Quigley EM, Fitzgerald GF, et al. Recombinant lactobacilli expressing linoleic acid isomerase can modulate the fatty acid composition of host adipose tissue in mice. Microbiology 2011; 157: 609-15.

27. Loguercio C, Federico A, Tuccillo C, Terracciano F, D'Auria MV, De Simone C, Del Vecchio Blanco C. Beneficial effects of a probiotic VSL\#3 on parameters of liver dysfunction in chronic liver diseases. J Clin Gastroenterol 2005; 39: 540-3.

28. Aller R, De Luis DA, Izaola O, Conde R, Gonzalez Sagrado M, Primo D, De La Fuente B, et al. Effect of a probiotic on liver aminotransferases in nonalcoholic fatty liver disease patients: a double blind randomized clinical trial. Eur Rev Med Pharmacol Sci 2011; 15: 1090-5.

29. McPherson S, Jonsson J R, Cowin GJ , O'Rourke P, Clouston $A D$, Volp $A$, Horsfall $L$, et al. Magnetic resonance imaging and spectroscopy accurately estimate the severity of steatosis provided the stage of fibrosis is considered. J Hepatol 2009; 51: 389-97.

30. Osman N, Adawi D, Molin G, Ahrne S, Berggren A, Jeppsson B. Bifidobacterium infantis strains with and without a combination of oligofructose and inulin (OFI) attenuate inflammation in DSS-induced colitis in rats. BMC Gastroenterol 2006; 6: 31

31. Osman N, Adawi D, Ahrne S, Jeppsson B, Molin G. Probiotics and blueberry attenuate the severity of dextran sulfate sodium (DSS)-induced colitis. Dig Dis Sci 2008; 53 : 2464-73.

32. Gabele E, Dostert K, Hofmann C, Wiest R, Scholmerich J, HeIlerbrand C, Obermeier F. DSS induced colitis increases portal LPS levels and enhances hepatic inflammation and fibrogenesis in experimental NASH. J Hepatol 2011; 55: 1391-9.

33. Henao-Mejia J, Elinav E, J in C, Hao L, Mehal WZ, Strowig T, Thaiss CA, et al. Inflammasome-mediated dysbiosis regulates progression of NAFLD and obesity. Nature 2012; 482: 179-85. 